\title{
DESIGN OF AFM CANTILEVERS FOR COMBINED THERMOMECHANICAL DATA WRITING AND READING
}

\author{
William P. King, Thomas W. Kenny, and Kenneth E. Goodson \\ Department of Mechanical Engineering, Stanford University \\ Stanford, California, 94305-3030 \\ Graham Cross, Michel Despont, Urs Dürig, Mark I. Lutwyche, Hugo Rothuizen, \\ Gerd K. Binnig, and Peter Vettiger \\ Micro- and Nanomechanics Group, IBM Research, Zurich Research Laboratory \\ CH-8803 Rüschlikon Switzerland
}

\begin{abstract}
A Joule-heated atomic force microscope (AFM) cantilever writes a data bit by scanning over a polymer substrate. Heat and force applied to the polymer cause it to soften and flow, yielding an indentation of radius near $50 \mathrm{~nm}$. Data is read by measuring the change in temperature of the heated cantilever as it follows the contour of an existing bit. Heat conduction governs the ultimate performance of a thermomechanical data storage device. The present work develops a finite-difference simulation of singlecantilever thermal and electrical behavior. Simulation results indicate design tradeoffs in power requirements, data writing speed, and data reading sensitivity. Scaling of single-cantilever predictions allows prediction of power requirements and ultimate data rates of cantilever arrays. The design tool predicts operating points for the present array cantilever, as well as for a proposed array cantilever. The framework established here considers for the first time comprehensive thermal, mechanical, and system-level requirements for data writing and reading in a thermomechanical data storage system.
\end{abstract}

\section{INTRODUCTION}

In today's magnetic data storage, the superparamagnetic effect limits the stability of the smallest magnetic domain, and will ultimately limit room-temperature magnetic storage technology at a data density of approximately $40 \mathrm{~Gb} / \mathrm{in}^{2}$ [1]. Several promising alternative data storage technologies that could surpass this limit involve the use of scanning-probe devices [2,3]. Thermomechanical data storage is one such technology [4-7].

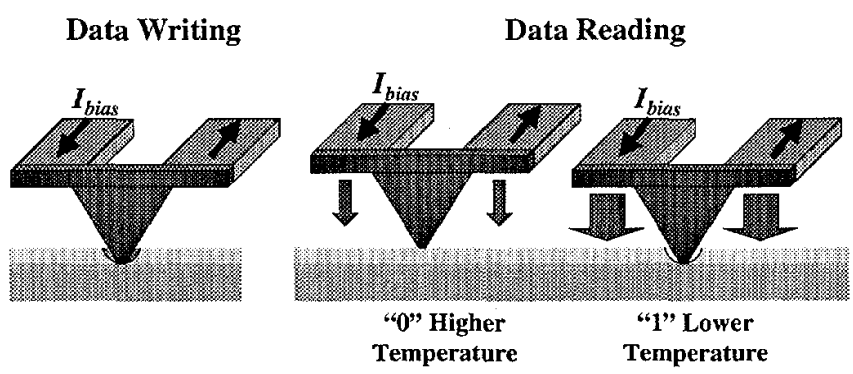

Figure 1. Thermomechanical data bit writing and thermal data reading with a Joule-heated AFM cantilever.

A Joule-heated AFM cantilever writes a data bit by scanning over a polymer substrate. Heat and force applied to the polymer cause it to soften and flow, thus writing a data bit, as shown in Fig. 1 [4-7]. Thermomechanical data storage with an electrically active silicon cantilever was first demonstrated to write data bits in a thick polycarbonate substrate with a cantilever thermal time constant of approximately $0.4 \mathrm{~ms}$ [4]. Silicon cantilevers with small solid-state Joule heaters near the cantilever tip were fabricated specifically for application to thermomechanical data storage [5]. This allowed cantilever optimization, which reduced the cantilever heating time into the sub-microsecond range [6].

Binnig et al. [7] wrote very small thermomechanical data bits on the order of $40 \mathrm{~nm}$ in diameter, shown in Fig. 2, in a PMMA layer of $40 \mathrm{~nm}$ thickness [7]. The very thin polymer layer confines the polymer melted-region to dimensions on the order of the polymer thickness, and limits tip penetration and therefore data bit size.

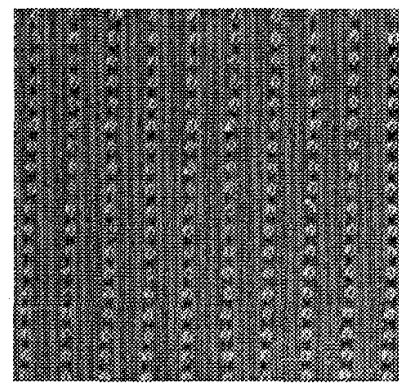

$150 \mathrm{~nm}-$

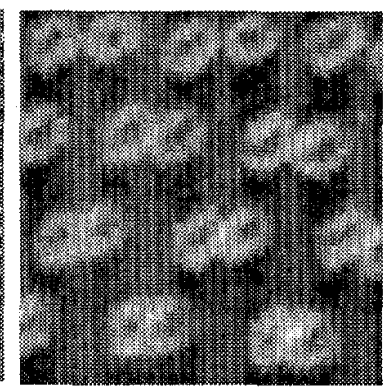

$40 \mathrm{~nm}-$
Figure 2. Tracks of thermomechanically written data bits (left) and bits written with high periodicity (right). The bit diameter and pitch of $40 \mathrm{~nm}$ corresponds to a data density of $0.4 \mathrm{~Tb} / \mathrm{in}^{2}$ [7].

Binnig et al. [7] also showed that existing pits can be located using the temperature signal from a heated cantilever. As the cantilever tip enters a previously written data bit, the reduction in thermal impedancc between the cantilcver and the substrate causes a lower temperature rise of the cantilever, as shown in Fig. 1. Measurement of the cantilever electrical resistance, which corresponds to cantilever temperature, determines the cantileversubstrate distance. The images in Fig. 2 were recorded by this thermal reading process.

In order to compete with traditional data storage technology, thermomechanical data storage must offer significant improvements over traditional data storage technology not only in data density but also in speed and power consumption. Array operation of heater-cantilevers allows higher data rates than a single cantilever configuration. Vettiger et al. [8] fabricated a $5 \times 5$ cantilever array, and used it to read topographical test structures with 200 $\mathrm{nm}$ features [9]. A $32 \times 32$ array of cantilevers was fabricated and wrote fields of data bits $[10,11]$. The most recent cantilever array is shown in Fig. 3. 


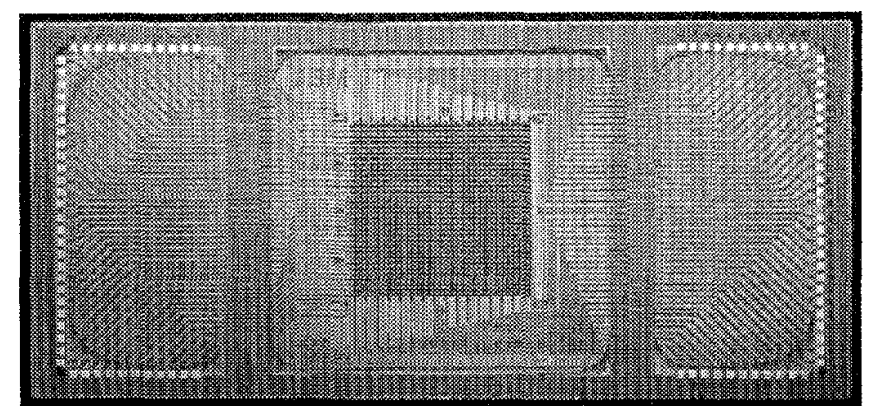

Figure 3. $32 \times 32$ (1024) array of cantilevers. From [12], (C) IEEE 1999.

While much progress has been made to decrease both the time to write a data bit and the size of the bit written, no work has been done to optimize cantilevers for thermal data reading. There has also been no work to design cantilevers for explicit array operation, or to identify tradeoffs in cantilever design. The present work aims to understand the heating and cooling of single AFM heater-cantilevers by measuring cantilever heating and cooling and through detailed device simulations. An electrical and thermal simulation is developed for the operation of a single cantilever and verified by measurements. Scaling of the single-cantilever predictions explores cantilever design and determines the operating window of the present cantilever array and one possible alternative design. Data rate is a key figure of merit in data storage, as well as power consumption in a possible mobile computing application. The present work aims to frame device operation in terms of these parameters.

\section{SINGLE-CANTILEVER MEASUREMENT AND SIMULATION}

Chui et al. [13] modeled and measured thermal and electrical behavior of heater-cantilevers for the purpose of studying thermal runaway. This approach employed a finite-difference simulation of heating in the cantilever. The present work develops a similar model, which improves upon the previous approach by accounting for heat transfer in the air near the cantilever, and into the underlying substrate. This improvement allows accurate time resolution as well as prediction of thermal data reading sensitivity.

Measurements were made on a cantilever having a design similar to the cantilevers from the array in Fig. 3 to verify the simulation. The measurement cantilever has a thickness of $1 \mu \mathrm{m}$, a length of $50 \mu \mathrm{m}$, a width of $5 \mu \mathrm{m}$, and a heater area of $5 \mu \mathrm{m} \times 5$ $\mu \mathrm{m}$. The cantilever is boron-doped with $1 \times 10^{17} \mathrm{~cm}^{-3}$ in the heater region and $1 \times 10^{20} \mathrm{~cm}^{-3}$ in the legs, yielding a total roomtemperature cantilever resistance of $924 \Omega$. The fabrication process for this cantilever is documented elsewhere [8]. The cantilever is placed in series with a sense resistor of $1 \mathrm{k} \Omega$, and voltage pulses are applied to the circuit. The measurement of the voltage across the circuit and the sense resistor allow the cantilever resistance to be calculated. Measurements of the cantilever resistance are made during square voltage pulses of $1 \mu \mathrm{s}, 10 \mu \mathrm{s}$, and $100 \mu$ s duration and a pulse amplitude of $1-15 \mathrm{~V}$. Following the heating pulse, a 1 $\mathrm{V}$ bias is applied to the cantilever in order to measure cantilever cooling.

The measurements and predictions here are made for a stationary cantilever in air. The model accounts for heat generation in the cantilever and for conduction into the air and along the cantilever. Scaling arguments show that thermal radiation and convection into the environment are small compared to conduction. The simulation parameters for the cantilever electrical properties and heater size geometry were used to fit the measured values. A model of the temperature-dependent intrinsic carrier generation in the cantilever is taken from Sze [14]. VLSI models calculate the temperature-dependent electrical resistivity of the doped silicon [15], and the thermal conductivity of the highly doped silicon cantilever is assumed to be $50 \mathrm{Wm}^{-1} \mathrm{~K}^{-1}[16]$.

Figure 4 shows the measurement and prediction of the maximum cantilever temperature for a range of voltage pulses. The simulated temperature compares well with measurements. The measured cantilever cooling, not shown here, also agrees well with simulations. The longer pulses indicate the nonlinearity of the electrical properties of the cantilever at higher temperature.

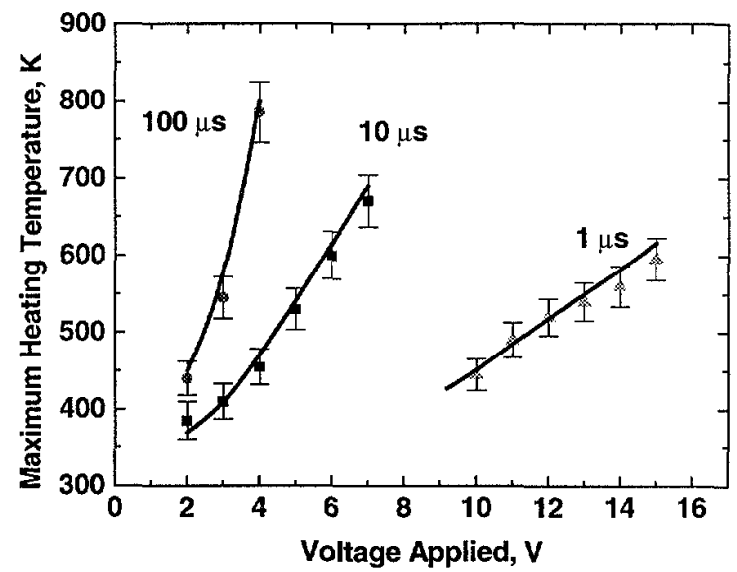

Figure 4. Measurement and prediction of maximum temperature during heating pulses of duration $1 \mu \mathrm{s}, 10 \mu \mathrm{s}$, and $100 \mu \mathrm{s}$. Data points represent measurements; solid lines represent prediction.

\section{SINGLE-CANTILEVER DESIGN AND ARRAY OPERATION}

This section describes predictions made for the operation of a cantilever from the array shown in Fig. 3 as well as the operation of a proposed cantilever. An SEM image of a cantilever from the array is shown in Fig. 5, as well as a diagram of the proposed cantilever. The design analysis presented here aims to explore data rate and related power requirements.

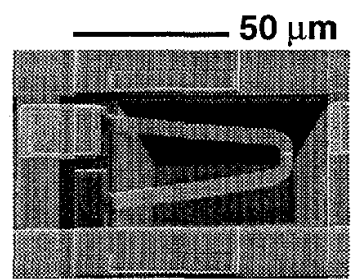

Present Cantilever

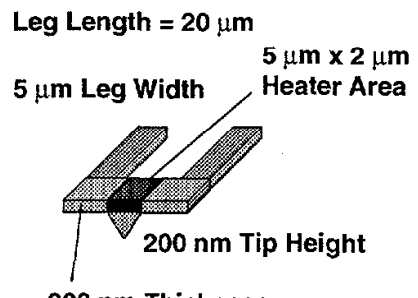

$200 \mathrm{~nm}$ Thickness
Figure 5. SEM image of a cantilever from the array shown in Fig. 3 , and a diagram of the proposed cantilever. The proposed cantilever could be fabricated with no additional fabrication requirements. The design details of both cantilevers pictured here are shown in Table 1. 
The simulations in this section employ the simulation tool used to make the predictions of cantilever heating shown in Fig. 4, with one modification: The simulations here model the cantilever near the polymer data surface, which is assumed to remain constant at room temperature. The maximum error induced by ignoring the temperature change in the substrate is estimated to be less than $5 \%$.

A single cantilever operates most efficiently if it is heated only long enough to reach the thermomechanical writing temperature. The thermomechanical writing temperature is taken to be $350{ }^{\circ} \mathrm{C}$, which is the measured writing temperature in thin PMMA [7,10]. In array operation, the cantilever can be selected again when it has cooled sufficiently, and moved to the appropriate position over the data media.

Table 1. Design details for present and proposed cantilevers.

\begin{tabular}{rcc}
\hline & $\begin{array}{c}\text { Present } \\
\text { Cantilever }\end{array}$ & $\begin{array}{c}\text { Proposed } \\
\text { Cantilever }\end{array}$ \\
\hline Heater Area & $5 \mu \mathrm{m} \times 7 \mu \mathrm{m}$ & $2 \mu \mathrm{m} \times 5 \mu \mathrm{m}$ \\
Thickness & $500 \mathrm{~nm}$ & $200 \mathrm{~nm}$ \\
Tip Height & $500 \mathrm{~nm}$ & $200 \mathrm{~nm}$ \\
Leg Width & $10 \mu \mathrm{m}$ & $5 \mu \mathrm{m}$ \\
Leg Length & $50 \mu \mathrm{m}$ & $20 \mu \mathrm{m}$ \\
Electrical Resistance & $2120 \Omega$ & $2012 \Omega$ \\
At 25 ${ }^{\circ} \mathrm{C}$ & & \\
Resonant Frequency & $220 \mathrm{kHz}$ & $330 \mathrm{kHz}$ \\
Mechanical Limit on & $968 \mathrm{kHz}$ & $1.45 \mathrm{MHz}$ \\
Repeated Data Writing & & \\
\hline
\end{tabular}

There are several constraints on cantilever design. It is desirable to minimize the power required to heat the cantilever with low operating voltage, which implies low electrical resistance. The electrical resistance of the cantilever heater region must however be much higher than any other element in the device. Successful thermal data reading has been accomplished for cantilevers having an electrical resistance in the range of $750 \Omega$ to $4 \mathrm{k} \Omega$. Reduction of the cantilever heater region size will generally reduce the cantilever thermal reading sensitivity. There is also a greater potential for electrical and thermal noise in cantilevers with very small heater regions.

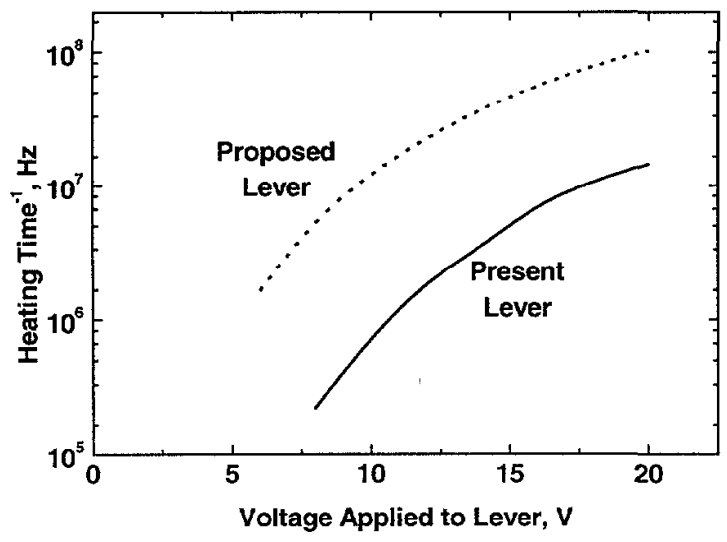

Figure 6. Predictions of the time required to reach the bit writing temperature as a function of voltage applied to the cantilever.
Figure 6 shows predictions for the time required for the cantilever to reach $350^{\circ} \mathrm{C}$, the measured writing temperature in PMMA. The predictions indicate that the lowest applied voltage at which the present cantilever can induce a data bit in a PMMA polymer layer is close to $8 \mathrm{~V}$, which agrees well with measurements.

Figure 7 shows the average power dissipated in the cantilever while writing a single data bit. These predictions show that the proposed cantilever does not have a significant reduction in power at a given applied voltage. However, predictions show that proposed cantilever could write bits at a lower minimum voltage and power than the present cantilever.

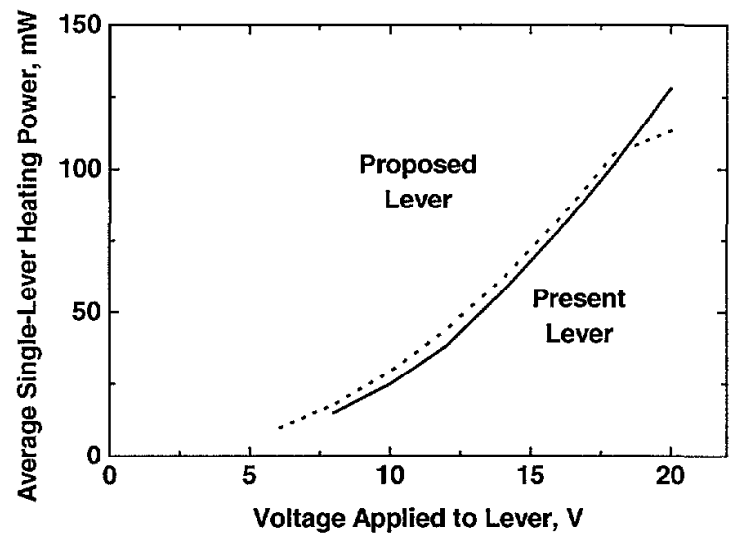

Figure 7. Predictions of average power required for a single cantilever to write a data bit as a function of voltage applied to the cantilever.

The thermal limit on the time in which a cantilever can repeatedly write data bits is governed by the time it takes for a cantilever to heat and then cool. The present work defines the cooling time as the time required for the cantilever to cool to onehalf its maximum heating temperature. Figure 8 shows predictions of the cantilever total thermal cycle time as a function of heating power. Although higher power generally corresponds to faster operation, there is an upper limit on the thermal cycle time that corresponds to the finite heat capacity of the cantilever heater region. At longer heating times, heat diffuses farther along the cantilever, increasing the time required to then cool the cantilever. This explains the strong dependence of the thermal cycle time on cantilever heating power at longer heating times.

Another limit on the time in which a cantilever can write successive data bits is the cantilever mechanical resonant frequency. In general, it is desirable to have a cantilever resonant frequency that is greater than the bit writing frequency. King et al. [17] showed that this is in fact not a strict requirement, as cantilevers in the array are not free to vibrate. Instead, the cantilevers are continuously forced against the polymer data layer, and penetrate only until the tip reaches the substrate beneath the thin polymer data layer. A more appropriate mechanical resonance figure of merit is the first harmonic of a cantilever with a hinged end, rather than a free end. The first resonance of a cantilever with a hinged end is approximately 4.4 times greater than the first resonance of a cantilever with a free end. The resonant frequency of a freely vibrating cantilever from the present array is approximately $220 \mathrm{kHz}$, and that of the proposed cantilever is estimated at $330 \mathrm{kHz}$. The appropriate values for the mechanical limit of repeated writing are $968 \mathrm{kHz}$ for the present cantilever and $1.45 \mathrm{MHz}$ for the proposed cantilever. 
The lowest possible power at which a thermomechanical data storage device could operate is of interest for mobile computing applications. At the lowest possible device power, each cantilever would be selected independent of other cantilevers, such that only one cantilever is heated at a time. In this case, the data storage device would operate at its lowest data rate.

The array in Fig. 3 is designed such that an entire row can read or write in concert, thus increasing data rate. The heating of an entire row of cantilevers at one time will increase the device power requirements. A possible data writing rate for multiplexed array operation is calculated in the following manner. The product of the cantilever heating time and the number of cantilever rows calculates the time for all the rows in the array to be selected once. The array cycle time must be greater than the total thermal cycle time of one row, as a cantilever must cool before it can move to a new location and write again. Another check must be made that the array cycle time is also greater than the cantilever mechanical response time. The number of cantilevers in the array is the number of bits that can be written in one array cycle.

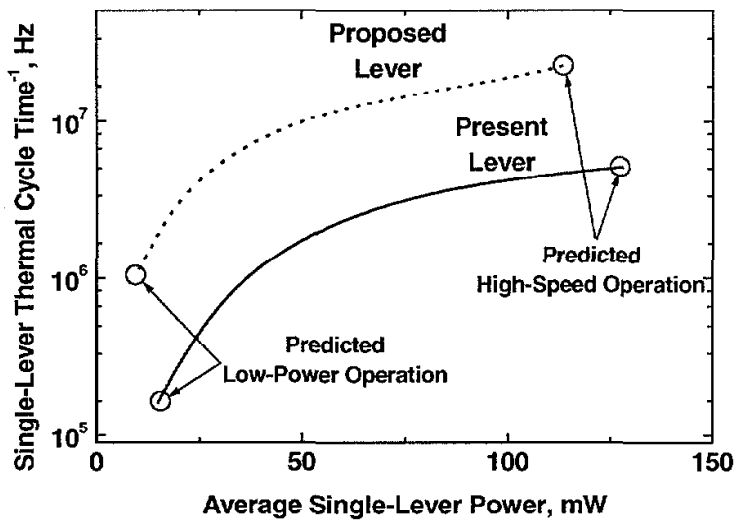

Figure 8. Single-cantilever total heating and cooling time as a function of applied power. The circles represent operating points for multiplexed cantilever arrays, listed in Table 2.

Table 2. Possible continuous-writing operation points for the present cantilever and proposed cantilever designs.

\begin{tabular}{|c|c|c|}
\hline & $\begin{array}{c}\text { Present } \\
\text { Cantilever }\end{array}$ & $\begin{array}{c}\text { Proposed } \\
\text { Cantilever }\end{array}$ \\
\hline Lowest Power & $0.3 \mathrm{Mb} / \mathrm{s}$ & $2.0 \mathrm{Mb} / \mathrm{s}$ \\
& $15.0 \mathrm{~mW}$ & $9.5 \mathrm{~mW}$ \\
\hline Highest Speed & $480 \mathrm{Mb} / \mathrm{s}$ & $10.0 \mathrm{~Gb} / \mathrm{s}$ \\
(Multiplexed) & $4.0 \mathrm{~W}$ & $13.0 \mathrm{~W}$ \\
& $32 \times 32 \mathrm{Array}$ & $100 \times 100$ Array \\
\hline
\end{tabular}

The circles in Fig. 8 are not data points, but represent possible operating points for continuous writing with an array of cantilevers. Table 2 describes these operation points in more detail. Two cases explore the boundaries of cantilever array operation, which are the highest possible data rate, and lowest possible power.

It is predicted that the present array could operate at its lowest possible power at $0.3 \mathrm{Mb} / \mathrm{s}$. This operation point does not exploit array multiplexing, and therefore the single-cantilever power determines the device power. It is predicted that low-power operation of an array of the proposed cantilevers would have a data rate of $2.0 \mathrm{Mb} / \mathrm{s}$ and a power requirement of $9.5 \mathrm{~mW}$.

I'he highest writing rate of the present cantilever array is governed by the time required for cantilever heating at $480 \mathrm{Mb} / \mathrm{s}$. Cantilever heating time also limits the fastest possible writing rate of a $100 \times 100$ array of proposed cantilevers at $10.0 \mathrm{~Gb} / \mathrm{s}$. Table 2 shows the increased power requirement of array operation. A $100 \times 100$ array consisting of the proposed cantilevers would have a device power requirement of $13 \mathrm{~W}$. It is important that a thermomechanical data storage device operated in this manner is cooled such that the power dissipated in the device does not melt the polymer data layer.

The power requirements and data rates presented here are for continuous writing. Arrays of cantilevers could operate such that they are continuously heated, and therefore do not need to be heated from room temperature for every bit-writing event. Also, data reading requires less power. Therefore typical device power for combination writing and reading operation could be substantially lower than the power required for continuous writing. We envision adaptive operation of a thermomechanical data storage device that could actively control where it operates on the power-speed curve.

Thermal data-reading sensitivity is calculated as the change in steady-state thermal impedance for a small change in cantilever tip height. The maximum thermal reading signal is obtained when the cantilever is heated to the highest temperature at which the polymer data surface is not modified, which is approximately $325^{\circ} \mathrm{C}[10]$. The cantilever power at this operation point is approximately $5 \mathrm{~mW}$. Predictions are shown in Fig. 8. The prediction of the sensitivity of the current cantilever array compares well with the measured value reported by Lutwyche et al. [10], also shown in Fig. 9.

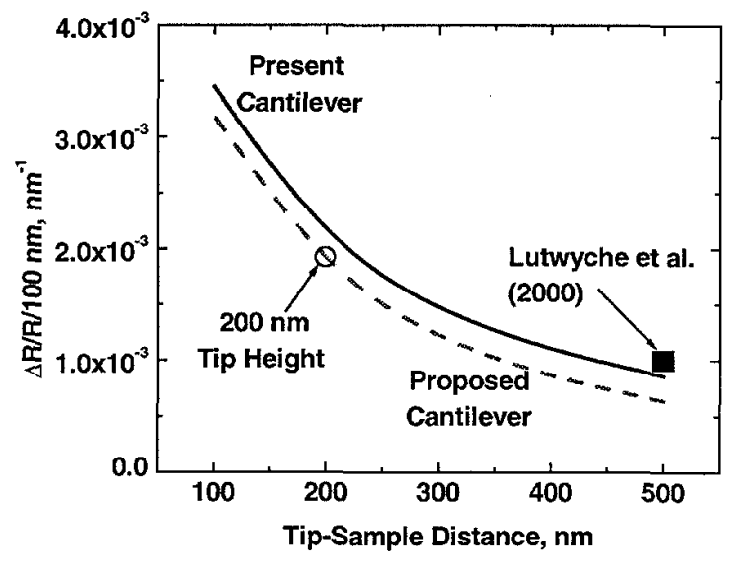

Figure 9. Sensitivity predictions for the current array cantilever and the proposed array cantilever. The sensitivity is calculated for reading a data bit of depth $100 \mathrm{~nm}$. The data represents the operating point of Lutwyche et al. [10].

In gencral, shorter and thinner cantilevers with smaller tips are more sensitive. While the proposed cantilever is thinner than the current cantilever, the predicted sensitivity of the proposed cantilever is slightly less than the present cantilever at a given cantilever-sample distance. This is due to the much smaller heater area and narrower legs of the proposed cantilever. It is predicted that a tip height of $200 \mathrm{~nm}$ for the proposed cantilever will result in a sensitivity of almost double that of the present cantilever, as shown in Fig. 8. 
Although the sensitivity calculation is made here at steady state, the result also indicates the transient value provided that two conditions are met: First, sufficient time must have elapsed for heat to diffuse across the cantilever-sample air gap, which is not necessarily the time required for the cantilever to reach steady state. For example, the time for heat to diffuse across a $200-\mathrm{nm}$ air gap is approximately $5 \mathrm{~ns}$. Secondly, the signal from the cantilever must be integrated over a sufficiently long time to achieve an acceptable signal-to-noise ratio. For transient sensing, we envision integrated read electronics fabricated directly on the cantilever array.

\section{CONCLUSIONS}

An electrical and thermal simulation of Joule-heated AFM cantilevers for thermomechanical data storage is developed and verified by comparison to measurements of cantilever heating. The simulation is then used to investigate the heating of the present array cantilever and a proposed array cantilever. Scaling of singlecantilever predictions allows prediction of array operation.

The framework established here considers for the first time comprehensive thermal, mechanical, and system-level requirements for data writing and reading in a thermomechanical data storage system. The approach developed herc is valid for polymers of various melting temperatures, and for thermal data writing and reading in other media.

\section{ACKNOWLEDGMENTS}

The authors gratefully acknowledge M. Asheghi, R. Beyeler, B. Chui, R. Germann, K. Kurabayashi, N. Mourlas, J. Santiago, P. Seidler and P. Sverdrup for discussions and support. U. Drechsler, G. Genolet, W. Häberle, R. Stutz, and R. Widmer of the IBM Zurich Micro- and Nanomechanics Group provided helpful assistance and advice. W.P.K. appreciates the support of IBM Research. K.E.G. was supported by an ONR Young Investigator Award.

\section{REFERENCES}

1. E. Grochowski and R. F. Hoyt, "Future Trends in Hard Disk Drives," IEEE Transactions on Magnetics, 32, 1850 (1996).

2. E. B. Cooper, S. R. Manalis, H. Fang, H. Dai, K. Matsumoto, S. C. Minnie, T. Hunt, and C. F. Quate, "Terabit-Per-Square-Inch Data Storage with the Atomic Force Microscope," Applied Physics Letters, 75, 3566 (1999).

3. H. J. Mamin, R. P. Ried, B. D. Terris, and D. Rugar, "High Density Data Storage Based on the Atomic Force Microscope," Applied Physics Letters, 87, 1014 (1999).

4. H. J. Mamin, "Thermal Writing Using a Heated Atomic Force Microscope Tip," Applied Physics Letters, 69, 433 (1996).

5. B. W. Chui, T. D. Stowe, T. W. Kenny, H. J. Mamin, B. D. Terris, and D. Rugar, "Low-Stiffness Silicon Cantilevers for Thermal Writing and Piezoresistive Readback with the Atomic Force Microscope," Applied Physics Letters, 69, 2767 (1996).

6. B. W. Chui, T. D. Stowe, Y. S. Ju, K. E. Goodson, T. W. Kenny, H. J. Mamin, B. D. Terris, R. P. Ried, and D. Rugar, "LowStiffness Silicon Cantilevers with Integrated Heaters and Piczoresistive Sensors for High-Density AFM Data Storage," Journal of Microelectromechanical Systems, 7, 69 (1998).
7. G. Binnig, M. Despont, U. Drechsler, W. Häberle, M. Lutwyche, P. Vettiger, H. J. Mamin, B. W. Chui, and T. W. Kenny, "Ultrahigh-Density Atomic Force Microscopy Data Storage with Erase Capability," Applied Physics Letters, 76, 1329 (1999).

8. P. Vettiger, J. Brugger, M. Despont, U. Drechsler, U. Dürig, W. Häberle, H. Rothuizen, R. Stutz, R. Widmer, and G. Binnig, "Ultrahigh Density, High-Data-Rate NEMS-Based AFM Data Storage System," Microelectronic Engineering, 46, 11 (1999).

9. M. Lutwyche, C. Andreoli, G. Binnig, J. Brugger, U. Drechsler, W. Häberle, H. Rohrer, H. Rothuizen, P. Vettiger, G. Yaralioglu, and C. Quate, "5 × 5 AFM Cantilever Arrays: A First Step Towards a Terabit Data Storage Device," Sensors and Actuators A, 73, 89 (1999).

10. M. I. Lutwyche, G. Cross, M. Despont, U. Drechsler, U. Dürig, W. Häberle, H. Rothuizen, R. Stutz, R. Widmer, G. K. Binnig, and P. Vettiger, "Millipede - A Highly-Parallel Dense ScanningProbe-Based Data Storage System", Technical Digest of IEEE International Solid-State Circuits Conference, San Francisco, CA (2000), pp. 126-127.

11. P. Vettiger, M. Despont, U. Drechsler, U. Dürig, W. Häberle, M. I. Lutwyche, H. Rothuizen, W. Stutz, W. Widmer, and G. Binnig, "The Millipede: More Than One Thousand Tips for Future AFM Data Storage," IBM Journal of Research and Development, in press.

12. M. Despont, J. Brugger, U. Drechsler, U. Dürig, W. Häberle, M. Lutwyche, H. Rothuizen, R. Stutz, R. Widmer, H. Rohrer, G. K. Binnig, and P. Vettiger, "VLSI-NEMS Chip for AFM Data Storage," Technical Digest $12^{\text {th }}$ IEEE International Micro Electro Mechanical Systems Conference MEMS '99, Orlando, FL, 01/99, IEEE, Piscataway (1999), pp. 564-569.

13. B. W. Chui, M. Asheghi, Y. S. Ju, K. E. Goodson, T. W. Kenny, and H. J. Mamin, "Intrinsic-Carrier Thermal Runaway in Silicon Microcantilevers," Microscale Thermophysical Engineering, 3. 217 (1999).

14. S. M. Sze, Physics of Semiconductor Devices, Wiley, New York (1981).

15. S. Selberherr, "Process and Device Modcling for VLSI," Microelectronics Reliability, 24, 225 (1984).

16. M. Asheghi, K. Kurabayashi, K. E. Goodson, R. Kasnavi, and J. Plummer, "Thermal Conduction in Doped Single-Crystal Silicon Films," Proceedings of the $33^{\text {rd }}$ National Heat Transfer Conference HTD99-186, Albuquerque, NM, 8/99, American Society of Mechanical Engineers, New York (1999), pp. 1-13.

17. W. P. King, J. G. Santiago, T. W. Kenny, and K. E. Goodson, "Modeling and Prediction of Sub-Micrometer Heat Transfer during Thermomechanical Data Storage," Proceedings of the ASME IMECE MEMS-Vol. I, Nashville, TN, 11/99, American Society of Mechanical Engineers, New York (1999), pp. 583-588. 